\title{
Multi-piconet Formation to Increase Channel Utilization in IEEE 802.15.3 High-Rate WPAN ${ }^{\star}$
}

\author{
Ssang-Bong Jung ${ }^{1}$, Soon-Bin Yim ${ }^{1}$, Tae-Jin Lee ${ }^{1}$, \\ Sun-Do June ${ }^{2}$, Hyeon-Seok Lee ${ }^{2}$, Tai-Gil Kwon ${ }^{2}$, and Jin-Woong Cho ${ }^{2}$ \\ ${ }^{1}$ School of Information and Communication Engineering, Sungkyunkwan University, Korea \\ ${ }^{2}$ Wireless Network Research Center, Korea Electronics Technology Institute, Korea \\ \{jssbong, sbyim, tjlee\}@ece.skku.ac.kr \\ $\{j$ sd, hslee75, tgkwon, chojw\}@keti.re.kr
}

\begin{abstract}
IEEE 802.15.3 high-rate Wireless Personal Area Networks (WPANs) is a short range communication technology, in which devices (DEVs) of a piconet communicate with a Piconet Coordinator (PNC). An extension of the communication area in WPAN via formation of a multi-piconet is one of the most challenging issues. Although a concept of multi-piconet has been defined in the IEEE 802.15.3 WPAN standard, a detailed formation mechanism of multi-piconet is not presented. In this paper, we propose efficient multi-piconet formation algorithms, Piconet Coordinator To Device (PCTD) and Device To Piconet Coordinator (DTPC). We evaluate the performance of the algorithms via simulations. The proposed algorithms are shown to extend the communication area with one to three levels and to provide sufficient Channel Time Allocations (CTAs) for subsidiary piconets in IEEE 802.15.3 WPAN.
\end{abstract}

\section{Introduction}

Recently, we have witnessed a noticeable increase of personal devices. The devices are often required to interact wirelessly to make information accessible and to exchange data without physical cables. Wireless Personal Area Networks (WPANs) can connect various personal devices within a Personal Operating Space (POS). IEEE 802.15.1 (Bluetooth) [1] and IEEE 802.15.4 (Zigbee) [3] supports the transmission rate of less than $1 \mathrm{Mbps}$, which is not appropriate for high-rate WPAN applications. IEEE 802.15.3 [2] [4] is an emerging high-rate WPAN technology, which enables portable multimedia devices to connect and communicate wirelessly. IEEE 802.15.3 WPAN operates in the unlicensed $2.4 \mathrm{GHz}$ ISM band with the data rate of up to 55Mbps and supports the ad-hoc mode allowing a number of devices to communicate with one another in a peerto-peer mode. A piconet in IEEE 802.15.3 consists of one Piconet Coordinator (PNC) and more than one device (DEV). In IEEE 802.15.3 WPAN, a multi-piconet can be formed to extend a single piconet. A multi-piconet consists of a parent piconet and its child piconets. The child PNC is a member of the parent piconet and also plays a role of the PNC in the child piconet. A child piconet is dependent on the parent piconet [5].

\footnotetext{
* This research was supported by Electro-0580 funded by the Ministry of Information and Communication (MIC) in South Korea. Dr. Tae-Jin Lee is the corresponding author.
} 
In the Bluetooth specification, a piconet consists of one master device and up to seven slave devices. A piconet can be extended in the form of a scatternet. A scatternet is a collection of multiple piconets and each piconet includes bridge devices to interconnect piconets. The formation of scatternets is essential to break the limit of a small piconet and allows interaction among Bluetooth piconets. There have been algorithms for scatternet formation, i.e., a distributed scatternet formation protocol [6], a formation mechanism to support device mobility [7], a Tree Scatternet Formation (TSF) algorithm [8], and the scatternet-route structure [9].

In the IEEE 802.15.3 specification, efficient multi-piconet formation protocols and Channel Time Allocation (CTA) mechanisms are not defined although basic frame structure for the multi-piconet extension is defined. A multi-piconet can extend the communication area of a single piconet. It is very important to form an interconnected network combining separate piconets and to provide new services to users. Therefore, in this paper, we propose an efficient multi-piconet formation scheme and evaluate its performance. In this paper, we present two multi-piconet formation algorithms, Piconet Coordinator-To-Device (PCTD) and Device-To-Piconet Coordinator (DTPC). In the PCTD multi-piconet formation algorithm, the formation of a multi-piconet is initiated by a PNC. The PNC allocates private CTAs to DEVs and the DEVs create child piconets to communicate one another in the allocated private CTAs. In the DTPC multi-piconet formation algorithm, the formation of a multi-piconet is initiated by some DEVs of a piconet and the PNC of the piconet allocates private CTAs to the DEVs. The initiating DEVs report the remote scan information to the PNC. The DEVs then create child piconts and communicate one another in the allocated private CTAs. An efficient private CTA allocation algorithm for PCTD and DTPC is also proposed. We conduct simulations to evaluate its performance. Simulation results validate the desired features of the PCTD and DTPC algorithms.

The rest of the paper is organized as follows. Section 2 gives an overview of the IEEE 802.15.3 High-rate WPAN. In Section 3, we describe our proposed PCTD and DTPC multi-piconet formation algorithms. Section 4 provides the performance analysis of the proposed algorithms. Finally, Section 5 concludes the paper.

\section{IEEE 802.15.3 High-Rate WPAN Overview}

In IEEE 802.15.3, a piconet consists of a PNC and more than one DEV. The PNC provides the basic timing for the piconet with beacons and it allows independent devices to communicate one another. The standard utilizes the $2.4 \mathrm{GHz}$ ISM unlicensed frequency band and two sets of channels are assigned for operation. The first set is the high-density mode which allocates 4 channels while the second one is the IEEE 802.11 b co-existence mode which allocates 3 channels. Since the two outer channels of the sets overlap, there are total of five channels.

In IEEE 802.15.3 WPAN, the channel time is divided into superframes, and each superframe begins with a beacon. Fig. 1 illustrates the MAC superframe structure. A superframe has three parts, i.e., beacon, Contention Access Period (CAP), and Channel Time Allocation Period (CTAP). The beacon is used to carry control and channel time allocation information to the entire piconet, and it is generated and broadcast by the 


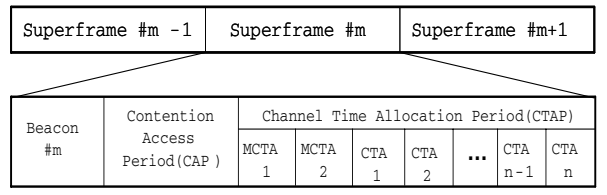

Fig. 1. Superframe structure of IEEE 802.15 .3

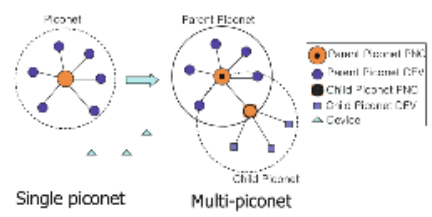

Fig. 2. Single piconet and multi-piconet

PNC. The CAP is used to exchange association request and response command, channel time request and response command, and asynchronous data. Carrier Sense Multiple Access/Collision Avoidance (CSMA/CA) is used for medium access control in the CAP. The CTAP is composed of channel time allocations (CTAs) and management CTAs (MCTAs), which makes TDMA-like medium access.

IEEE 802.15.3 WPAN allows a DEV to request the formation of a subsidiary piconet. There are subsidiary piconets of two types. One is the child piconet and the other one is the neighbor piconet. The original piconet is referred to as the parent piconet. The neighbor piconet can not communicate with the parent piconet, but the child piconet can communicate with the parent piconet. We call a multi-piconet as a larger piconet consisting of a parent piconet and its child piconets. Fig. 2 shows a single piconet and a multi-piconet. A parent piconet and its child piconets operate under the same channel. A child PNC requests private CTAs to the parent PNC and the parent PNC allocates private CTAs to the child PNC. A child piconet can then communicate in the private CTA period.

\section{Proposed Multi-piconet Formation Algorithms}

In this section, we present our proposed two multi-piconet formation algorithms to extend limited piconet communication area. One is the PCTD multi-piconet formation algorithm and the other one is the DTPC multi-piconet formation algorithm.

\subsection{PCTD Multi-piconet Formation Algorithm}

In this section, we describe the PCTD multi-piconet formation algorithm. In the PCTD algorithm, a PNC starts to form a multi-piconet, empowers some DEVs to be child piconet PNCs and allocates private CTAs to the DEVs. The DEVs which are assigned the private CTAs have the PNC capability. The PCTD multi-piconet formation procedure is shown in Fig. 3.

If a parent PNC has its available capacity to distribute and there exist DEVs in the current piconet, the PNC selects some DEVs as child PNCs by the child PNCs selection (CPS) algorithm shown in Fig. 4. Then, the PNC allocates private CTAs to the selected DEVs by Eqs. (1) (5). The selected DEVs make piconets, i.e., child piconets. Child piconets can now communicate in the allocated private CTAs. The mechanism of selecting some DEVs as child PNCs works as follows. If the number of DEVs in a piconet is less than the minimum number of DEVs (min_DEVs), we select all the DEVs. 


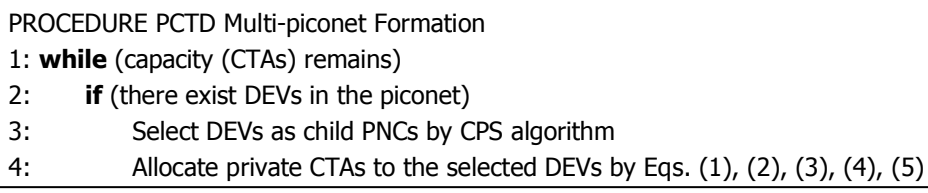

Fig. 3. PCTD multi-piconet formation algorithm

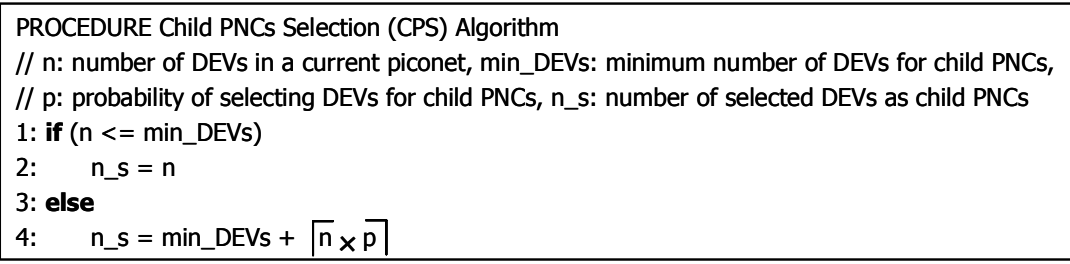

Fig. 4. Child PNCs Selection (CPS) algorithm

Otherwise, we randomly select some DEVs among the DEVs with the probability of $p$ while providing the minimum number of DEVs.

Allocation of private CTAs is done as follows based on the basic capacity for level $i$, $C_{\text {capacity }}^{i}$ and the allocation CTA remainder to one piconet for level $i, R_{C T A l}^{i}$. To explain the private CTA allocation algorithm for the PCTD multi-piconet formation algorithm, we define the following notations:

$-T_{B}:$ Beacon time

- $T_{C A P}:$ Channel access period

- $T_{C T A}$ : Channel time allocation

- $S$ : Maximum superframe size

$-N$ : Number of DEVs in the parent piconet

- $L$ : Number of levels

- $n_{i}$ : Number of DEVs in level $i, \quad i=1,2, \cdots, L$

- $m_{i}$ : Number of child piconets in level $i, \quad i=1,2, \cdots, L$

- $R_{C T A 1}^{i}$ : CTA allocation remainder to one piconet in level $i, \quad i=1,2, \cdots, L$

- $C_{\text {capacity }}^{i}$ : Basic capacity for level $i, \quad i=1,2, \cdots, L$

- $C_{\text {capacity }}^{0}$ : Basic capacity for level 0 (parent piconet)

- $C_{\text {max }}^{i}$ : Maximum capacity for level $i, \quad i=1,2, \cdots, L$

The $C_{\text {capacity }}^{0}$ consists of a beacon time and a CAP, and the basic CTAs for the parent piconet DEVs and is computed as Eq. (1). We can calculate the basic capacity for level $i, C_{\text {capacity }}^{i}$ by Eq. (2).

$$
\begin{aligned}
& C_{\text {capacity }}^{0}=\left(T_{B}+T_{C A P}\right)+\left(T_{C T A} \times N\right) \\
& C_{\text {capacity }}^{i}=\left(T_{B}+T_{C A P}\right) \times m_{i}+\left(T_{C T A} \times n_{i}\right), \quad i=1,2, \cdots, L
\end{aligned}
$$




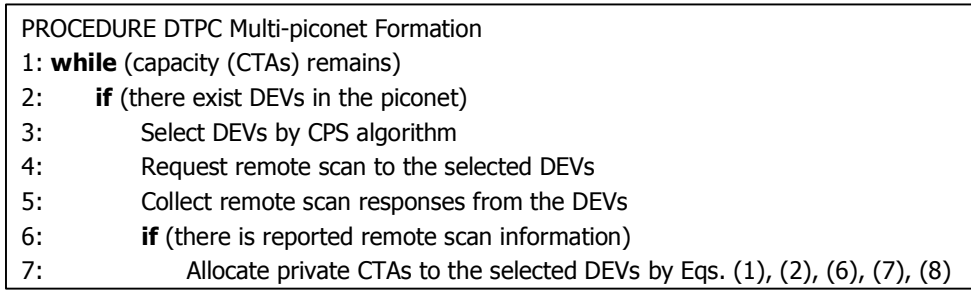

Fig. 5. DTPC multi-piconet formation algorithm

since $m_{i}$ child piconets require beacons and CAPs and $n_{i}$ DEVs in level $i$ are assigned $T_{C T A}$. The remaining capacity has to be allocated for the parent piconet and its child piconets. It the PCTD algorithm, the information on the next level is not known, the CTA allocation remainder to one child piconet for level 1 is found as follows:

$$
R_{C T A 1}^{l}=\frac{S-C_{\text {capacity }}^{0}}{m_{1}+1}
$$

since $S-C_{\text {capacity }}^{0}$ is the remaining capacity for level 1 and $m_{l}$ is number of child piconets in level 1 . Note that $C_{\text {capacity }}^{0}$ does not contain $n_{l}$, which is the information on the next level. The 1 in $\left(m_{1}+1\right)$ denotes the parent piconet. Similarly, we can find the CTA allocation remainder in level $i, R_{C T A I}^{i}$ as follows.

$$
R_{C T A 1}^{i}=\frac{R_{C T A 1}^{i-1} \times m_{i-1}-C_{\text {capacity }}^{i-1}}{m_{i}+m_{i-1}}, \quad i=2,3, \cdots, L .
$$

Thus, the private CTAs for each child piconet is equal to $R_{C T A I}^{i}$ in the PCTD multipiconet formation algorithm. The maximum capacity at level $i, C_{\max }^{i}$ is given by

$$
C_{\text {max }}^{i}=R_{C T A 1}^{i} \times m_{i}, \quad i=1,2, \cdots, L
$$

from the fact that each child piconet of level $i$ is assigned $R_{C T A l}^{i}$ and there are $m_{i}$ child piconets.

\subsection{DTPC Multi-piconet Formation Algorithm}

In this section, we describe the DTPC multi-piconet formation algorithm. In the DTPC algorithm, a PNC requests remote scan to some selected DEVs. The selected DEVs respond remote scan information to the PNC. The PNC then allocates private CTAs to the DEVs. The DTPC multi-piconet formation procedure is shown in Fig. 5. First, DEVs make their own piconets or join already formed piconets. The PNC of a piconet requests remote scan to some selected DEVs and the selection is done by the CPS algorithm, and the DEVs respond remote scan information with the near piconets to the PNC. The DEVs then request private CTAs to the PNC to form child piconets. The PNC allocates private CTAs to the requesting DEVs by Eqs. (1), (2), (6), (7), (8) and the requesting DEVs make child piconets. 


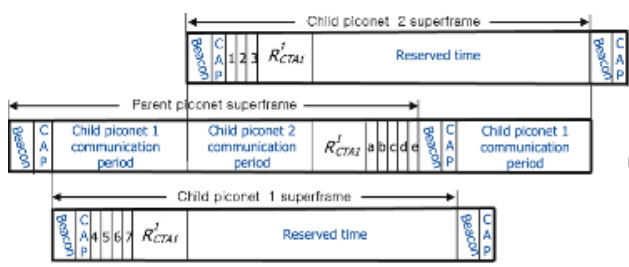

(a)

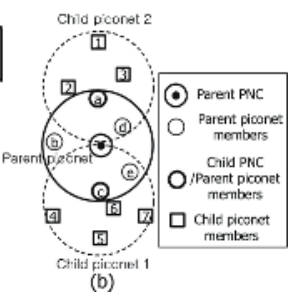

Fig. 6. Multi-piconet CTA allocation example (a - e: parent picinet DEVs, 1 - 7: child piconet DEVs), (a) superframe allocation to child piconets (b) a multi-piconet topology

The basic capacity at level 0 (parent piconet) $C_{\text {capacity }}^{0}$ and the basic capacity at level $i C_{\text {capacity }}^{i}$ are the same as in Eqs. (1) and (2). The CTA allocation remainder at level 1, $R_{C T A l}^{l}$ and the CTA allocation remainder at level $i, R_{C T A l}^{i}$ are calculated as follows:

$$
\begin{aligned}
R_{C T A 1}^{l} & =\frac{S-\left(C_{\text {capacity }}^{l}+C_{\text {capacity }}^{0}\right)}{m_{1}+1} \\
R_{C T A 1}^{i} & =\frac{R_{C T A 1}^{i-1} \times m_{i-1}-C_{\text {capacity }}^{i}}{m_{i}+m_{i-1}}, \quad i=2,3, \cdots, L
\end{aligned}
$$

since $S-\left(C_{\text {capacity }}^{l}+C_{\text {capacity }}^{0}\right)$ is the remaining capacity for level 1 and $m_{l}$ is the number of child piconets in level 1 . The 1 in $\left(m_{l}+1\right)$ denotes the parent piconet. In the PCTD algorithm the information on the next level is not known, we find $R_{C T A 1}^{i}$ by Eqs. (3) and (4). However, in the DTPC algorithm, we can know the information on the next level. Therefore, $R_{C T A 1}^{l}$ and $R_{C T A l}^{i}$ in DTPC algorithm is found as Eqs. (6) and (7).

The maximum capacity of level $i, C_{\max }^{i}$ is given by

$$
C_{\text {max }}^{i}=R_{C T A 1}^{i} \times m_{i}+C_{\text {capacity }}^{i}, \quad i=1,2, \cdots, L
$$

from the fact that each child piconet of level $i$ is assigned $R_{C T A 1}^{i}$ and there are $m_{i}$ child piconet, and $C_{\text {capacity }}^{i}$ is the basic capacity of level $i$.

Fig. 6 (b) shows a multi-piconet with level $L=1$ and the number of child piconets $m_{1}=2$. Fig. 6 (a) shows the relationship of superframes for the topology. The superframe of a parent piconet consists of the beacon, CAP, the communication period for the child piconets, the local communication period for the parent piconet DEVs (a, b, c, d, e) and the CTA allocation remainder $R_{C T A I}^{l}$. The communication period of child piconet 1 is 4 , $5,6,7$ and the communication period of child piconet 2 is $1,2,3$. The CTA allocation remainder $\left(R_{C T A 1}^{l}\right)$ is required to reserve communications between the parent piconet and child piconets, and to make child piconets of the next level.

\section{Performance Evaluation}

We evaluate the performance of the proposed two multi-piconet formation algorithms via simulations. The simulation parameters are summarized in Table 1 . The DEVs are assumed to be located uniformly in a square area of $50 \mathrm{~m} \times 50 \mathrm{~m}$ and $60 \mathrm{~m} \times 60 \mathrm{~m}$. And 
Table 1. Simulation parameters

\begin{tabular}{||c|c||}
\hline Parameter & Value \\
\hline \hline Channel bandwidth & $11 \mathrm{Mbps}$ \\
\hline Total number of DEVs & $10 \sim 150$ \\
\hline CTA size & $2,048 \mathrm{byte}$ \\
\hline CTA time $\left(T_{C T A}\right)$ & $1.53 \mathrm{~ms}$ \\
\hline Beacon time $\left(T_{B}\right)$ & $0.06 \mathrm{~ms}$ \\
\hline CAP time $\left(T_{C A P}\right)$ & $1.00 \mathrm{~ms}$ \\
\hline Maximum Superframe size $(S)$ & $65.535 \mathrm{~ms}$ \\
\hline Minimum number of DEVs $(\min D E V s)$ & 4 \\
\hline Probability to select DEVs $(p)$ & 0.2 \\
\hline
\end{tabular}

the number of DEVs ranges from 10 to 150 in the area. The number of simulation runs is 500. The length of a MAC Service Data Unit (MSDU) is set to 2048 bytes, which equals to the unit CTA size 1 . We analyze the performance of the PCDT and the DTPC multi-piconet formation algorithm with respect to the number of formed multi-piconet levels and the remaining capacity.

We then analyze the performance for random networks. Fig. 7 and Fig. 8 present the formed multi-piconet levels by the proposed algorithms. The PCTD algorithm is shown to form multi-piconets with two to three levels, and the DTPC algorithm is shown to form multi-piconets with one to two levels. The proposed algorithms form more levels of a multi-piconet if the density of DEVs is high and the PCTD algorithm generates more levels than the DTPC algorithm does. With the proposed algorithms, one can extend the communication range two to three times. In particular, the communication range of a formed multi-piconet by the PCTD algorithm is extended up to 50m assuming the transmission range of a DEV is $10 \mathrm{~m}$. The PCTD algorithm exhibits higher efficiency than the DTPC algorithm. We define the efficiency as the ratio between the allocated capacity and the original total capacity. For example, in Fig. 7, if the number of DEVs is 70 , the PCDT algorithm is $130 \%$ better than the DTPC algorithm in terms of the formation efficiency. And, if the number of DEVs is 110, the PCDT algorithm is $114 \%$ better than the DTPC algorithm.

We also analyze the remaining capacity at the final level. Fig. 9 and Fig. 10 present the remaining multi-piconet capacity as the number of DEVs varies. If the number of DEVs increases, the remaining capacity decreases. Since the maximum superframe size is $65.535 \mathrm{~ms}$, multi-piconet formation is possible up to level 3 . Thus, the more the number of DEVs, the higher the level of a multi-piconet becomes and the less the remaining capacity of the last level becomes.

In the PCTD algorithm, if the capacity remains to make child piconets, it can directly form new child piconets. However, in the DTPC algorithm, although the capacity remains to make child piconets, if the near piconet information as a result of remote scan does not exist, it can not make new child piconets. Thus, the remaining capacity at the last level in PCTD is smaller than that in DTPC as in Fig. 9 and Fig. 10. And the level of the PCTD algorithm is higher than that of the DTPC algorithm.

\footnotetext{
${ }^{1}$ In fact, physical and MAC header and preambles are appended as well.
} 


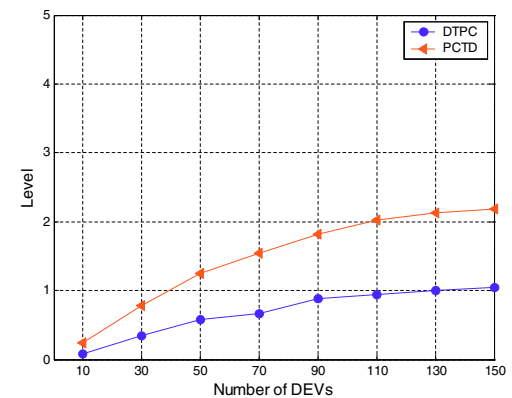

Fig. 7. Multi-piconet level as the number of DEVs $(50 \mathrm{~m} \times 50 \mathrm{~m})$ varies

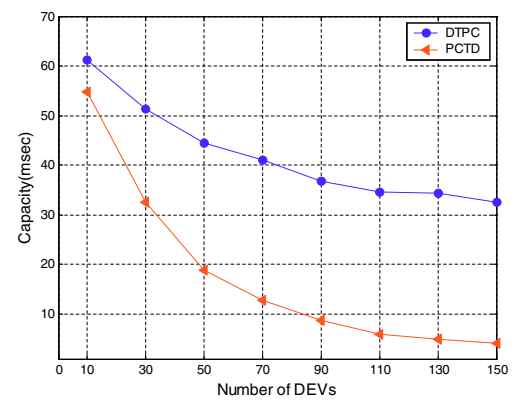

Fig. 9. Remaining multi-piconet capacity as the number of DEVs $(50 \mathrm{~m} \times 50 \mathrm{~m})$ varies

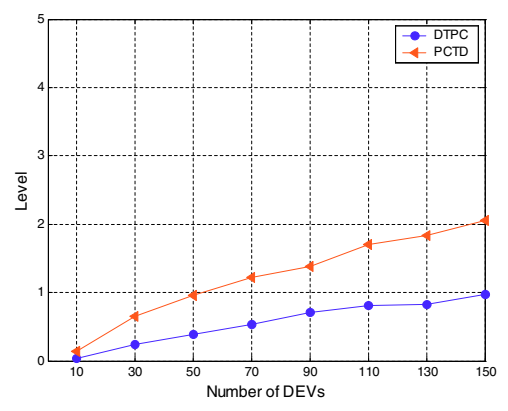

Fig. 8. Multi-piconet level as the number of DEVs $(60 \mathrm{~m} \times 60 \mathrm{~m})$ varies

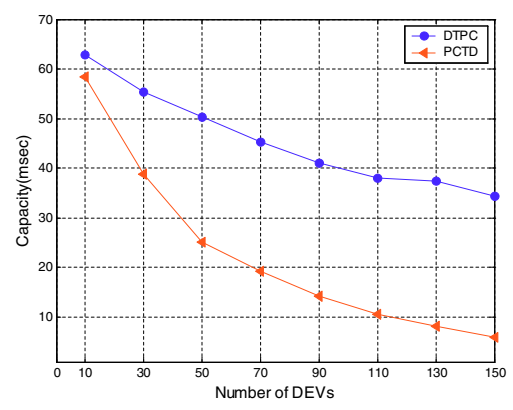

Fig. 10. Remaining multi-piconet capacity as the number of DEVs $(60 \mathrm{~m} \times 60 \mathrm{~m})$ varies

\section{Conclusion}

In this paper, we have proposed the multi-piconet formation mechanisms with efficient private CTA allocation schemes to extend the piconet communication area of IEEE 802.15.3 WPAN. We evaluate the performance of the proposed algorithms via simulations. The results show the relationships of the level, the capacity and the number of DEVs for multi-piconet formation. The PCTD algorithm is shown to form multipiconets with two to three levels, but the available capacity at the last level for child piconets is limited. The DTPC algorithm can form multi-piconets with one to two levels, and the available capacity at the last level for child piconets is sufficient. The proposed algorithms are able to extend the communication area and to generate large-scale WPAN service.

\section{References}

1. IEEE, "Standards for Part 15.1: Wireless Medium Access Control (MAC) and Physical Layer (PHY) Specifications for Wireless Personal Area Networks (WPANs),” Jun. 2002.

2. IEEE, "Standard for Part 15.3: Wireless Medium Access Control (MAC) and Physical Layer (PHY) Specifications for High Rate Wireless Personal Area Networks (WPANs), ' Sep. 2003. 
3. IEEE, "Standard for Part 15.4: Wireless Medium Access Control (MAC) and Physical Layer (PHY) Specifications for Low-Rate Wireless Personal Area Networks (LR-WPANs)," Oct. 2003.

4. J. Karaoguz, "High-Rate Wireless Personal Area Networks," IEEE Communications Magazine, vol. 39, no. 12, pp. 96-102, Dec. 2001.

5. D. Trezentos, G. Froc, I. Moreau and X. Lagrange, "Algorithms for Ad-hoc Piconet Topology Initialization Evaluation for the IEEE 802.15.3 High Rate WPAN System," in Proc. of IEEE VTC, Vol. 5, pp. 3448-3452, Oct. 2003.

6. V. P. Verma and A. A. Chandak, "Distributed Bluetooth Scatternet Formation Algorithm," in Proc. of IEEE GLOBECOM, vol. 3, pp. 1274-1278, Dec. 2003.

7. C. H. Yang and Y. S. Chen, "Bluetooth Scatternet Formation for Supporting Device Mobility," in Proc. of IEEE ITRC, pp. 408-412, Jun. 2005.

8. G. Zaruba, S. Basagni, and I. Chlamtac, "Bluetooth-Scatternet Formation to Enable Bluetooth Based Ad Hoc Networks," in Proc. of IEEE ICC, vol. 1, pp. 273-277, Jun. 2001.

9. Y. Liu, M. Lee and T. Saadawi, "A Bluetooth Scatternet-Route Structure for Multihop Ad Hoc Networks,” IEEE J. on Set. Areas in Communications, vol. 21, no. 2, pp. 229-239, Feb. 2003. 